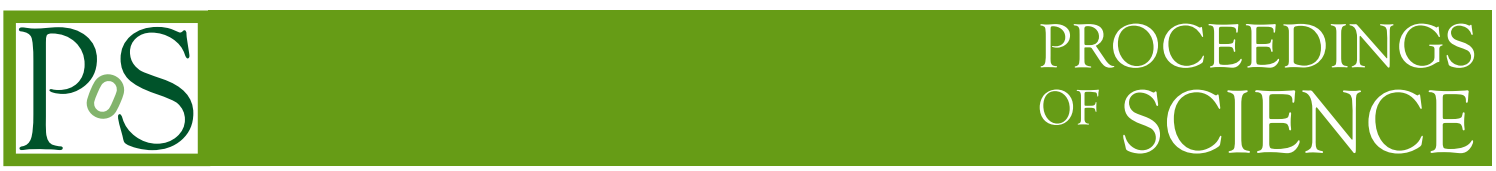

\title{
The Cygno Experiment
}

F.D. Amaro, ${ }^{a}$ E. Baracchini, ${ }^{b, c}$ L. Benussi, ${ }^{d}$ S. Bianco, ${ }^{d}$ C. Capoccia, ${ }^{d}$ M. Caponero, ${ }^{d, e}$ D.S. Cardoso, ${ }^{f}$ G. Cavoto, ${ }^{g, h, *}$ A. Cortez, ${ }^{b, c}$ I.A. Costa, ${ }^{i}$ G. D'Imperio, ${ }^{h}$ E. Dané, ${ }^{d}$ G. Dho, ${ }^{b, c}$ F. Di Giambattista, ${ }^{b, c}$ E. Di Marco, ${ }^{h}$ F. lacoangeli, ${ }^{h}$ H.P. Lima Júnior, ${ }^{f}$ A.d.S. Lopes Júnior, ${ }^{i}$ G. Maccarrone, ${ }^{d}$ R.D.P. Mano, ${ }^{j}$ M. Marafini, ${ }^{k}$ R.R. Marcelo Gregorio, ${ }^{l}$ D.J.G. Marques, ${ }^{b, c}$ G. Mazzitelli, ${ }^{d}$ A.G. McLean, ${ }^{l}$ A. Messina, ${ }^{g}, h$ C.M.B. Monteiro, ${ }^{j}$ R.A. Nobrega, ${ }^{i}$ I.F. Pains, ${ }^{i}$ E. Paoletti, ${ }^{d}$ L. Passamonti, ${ }^{d}$ S. Pelosi, ${ }^{h}$ F. Petrucci, ${ }^{m}$ S. Piacentini, ${ }^{g, h}$ D. Piccolo, ${ }^{d}$ D. Pierluigi, ${ }^{d}$ D. Pinci, ${ }^{h}$ A. Prajapati, ${ }^{b, c}$ F. Renga, ${ }^{h}$ R.C. Roque, ${ }^{j}$ F. Rosatelli, ${ }^{d}$ A. Russo, ${ }^{d}$ J.M.F.d. Santos, ${ }^{j}$ G. Saviano, ${ }^{d, n}$ N.J.C. Spooner, ${ }^{l}$ R. Tesauro, ${ }^{d}$ S. Tomassini ${ }^{d}$ and S. Torelli ${ }^{b, c}$

${ }^{a}$ Department of Physics, University of Coimbra, 3004-516 Coimbra, Portugal

${ }^{b}$ Gran Sasso Science Institute, 67100, L’Aquila, Italy

${ }^{c}$ Istituto Nazionale di Fisica Nucleare, Laboratori Nazionali del Gran Sasso, 67100, Assergi, Italy

${ }^{d}$ Istituto Nazionale di Fisica Nucleare, Laboratori Nazionali di Frascati, 00044, Frascati, Italy

${ }^{e}$ ENEA Centro Ricerche Frascati, 00044, Frascati, Italy

${ }^{f}$ Centro Brasileiro de Pesquisas Físicas, Rio de Janeiro 22290-180, RJ, Brazil

${ }^{g}$ Dipartimento di Fisica, Università La Sapienza di Roma, 00185, Roma, Italy

${ }^{h}$ Istituto Nazionale di Fisica Nucleare, Sezione di Roma, 00185, Rome, Italy

${ }^{i}$ Universidade Federal de Juiz de Fora, Faculdade de Engenharia, 36036-900, Juiz de Fora, MG, Brasil

${ }^{j}$ LIBPhys, Department of Physics, University of Coimbra, 3004-516 Coimbra, Portugal

${ }^{k}$ Museo Storico della Fisica e Centro Studi e Ricerche "Enrico Fermi”, Piazza del Viminale 1, 00184, Roma, Italy

${ }^{l}$ Department of Physics and Astronomy, University of Sheffield, Sheffield, S3 7RH, UK

${ }^{m}$ Dipartimento di Matematica e Fisica, Università Roma TRE, 00146, Roma, Italy

${ }^{n}$ Dipartimento di Ingegneria Chimica, Materiali e Ambiente, Sapienza Università di Roma, 00185, Roma, Italy

E-mail: gianluca.cavoto@roma1.infn.it

\footnotetext{
${ }^{*}$ Speaker
} 
The detection of ultra-rare events as the interaction of galactic dark matter (DM) candidate particles or of neutrinos originated from the Sun requires the development of innovative detection techniques. In particular future experiments for direct DM detection requires to extend their sensitivity to masses well below $10 \mathrm{GeV}$.

The Cygno collaboration plans to build and operate at the Laboratori Nazionali del Gran Sasso (LNGS) a cubic meter demonstrator of a gaseous time projection chamber (TPC), equipped with an optical readout and using a He:CF4 gas mixture kept at atmospheric pressure. The presence of low $\mathrm{Z}$ atoms allows to reach a competitive sensitivity to DM masses in the $\mathrm{GeV}$ range while the presence of fluorine can be used to set limits on a spin-dependent DM interaction cross-section.

The Cygno TPC is equipped with a Gas Electron Multipliers (GEM) amplification stage of the primary ionization electrons. Light is produced from the GEM while scientific CMOS cameras and fast photodetectors are combined to obtaining a three-dimensional reconstruction of the tracks either due to nuclear or to electron recoils. The design and the sensitivity of the demonstrator based on advanced Monte Carlo simulations including the radioactivity of the materials and of the LNGS cavern are reported. Pattern recognition algorithms are used to evaluate the identification capability of nuclear recoils against electronic recoils and studied in data from small scale prototypes. Energy measurement and also sensitivity to the source directionality are also evaluated. A Cygno TPC would therefore be sensitive to the direction of electron recoils originated by solar neutrinos interactions. The Cygno collaboration plans to demonstrate the scalability of such detector concept to reach a target mass large enough to significantly extend our knowledge about DM nature and solar neutrinos.

\footnotetext{
*** The European Physical Society Conference on High Energy Physics (EPS-HEP2021), *** *** 26-30 July $2021 * * *$

*** Online conference, jointly organized by Universität Hamburg and the research center DESY ***
} 


\section{Gas detectors for dark matter}

The detection of dark matter (DM) particles from the Galactic halo is based on the assumption that DM can elastically scatter on a nucleus of a sensitive material in a detector. The nucleus is then recoiling with a usually faint kinetic energy (down to the $\mathrm{keV}$ range). A process with similar kinetic energy can be initiated by solar neutrinos inducing either nuclear or atomic electrons recoils (NR and ER, respectively).

Most of the DM detectors currently in operation feature massive targets where the recoil energy is transformed into scintillation light, heat or charge in an extremely small surrounding of the interaction point. These targets are usually dense and therefore DM detectors are relatively compact. On the contrary a time projection chamber (TPC) using a gas mixture can record a mm-sized track due to the ER or NR. This is due to the low density of gas and therefore NR can be distinguished by ER thanks to the different energy loss density and the direction of the incoming DM particles can be inferred - adding therefore a new tool to study the presence of DM in our Galaxy. Clearly a large volume should be employed in view of a future experimental program and therefore a scaling of the detection technology is needed at a relatively low cost to instrument tons of target material [1].

The Cygno experiment is aiming to study the optical readout of a gas TPC [2-5]. In the sensitive volume the charged particles produce a trail of electron clusters that are then drifted by a $1 \mathrm{kV} / \mathrm{cm}$ electric field to the anode. The anode is instrumented with a triple GEM (Gas electron multiplier) system that generates an avalanche where visibile light is produced along with charge. This light is then focused onto a scientific CMOS camera with single photon sensitivity. In Fig. 1 an image of cosmic rays crossing the gas volume of one of the Cygno prototypes is shown.
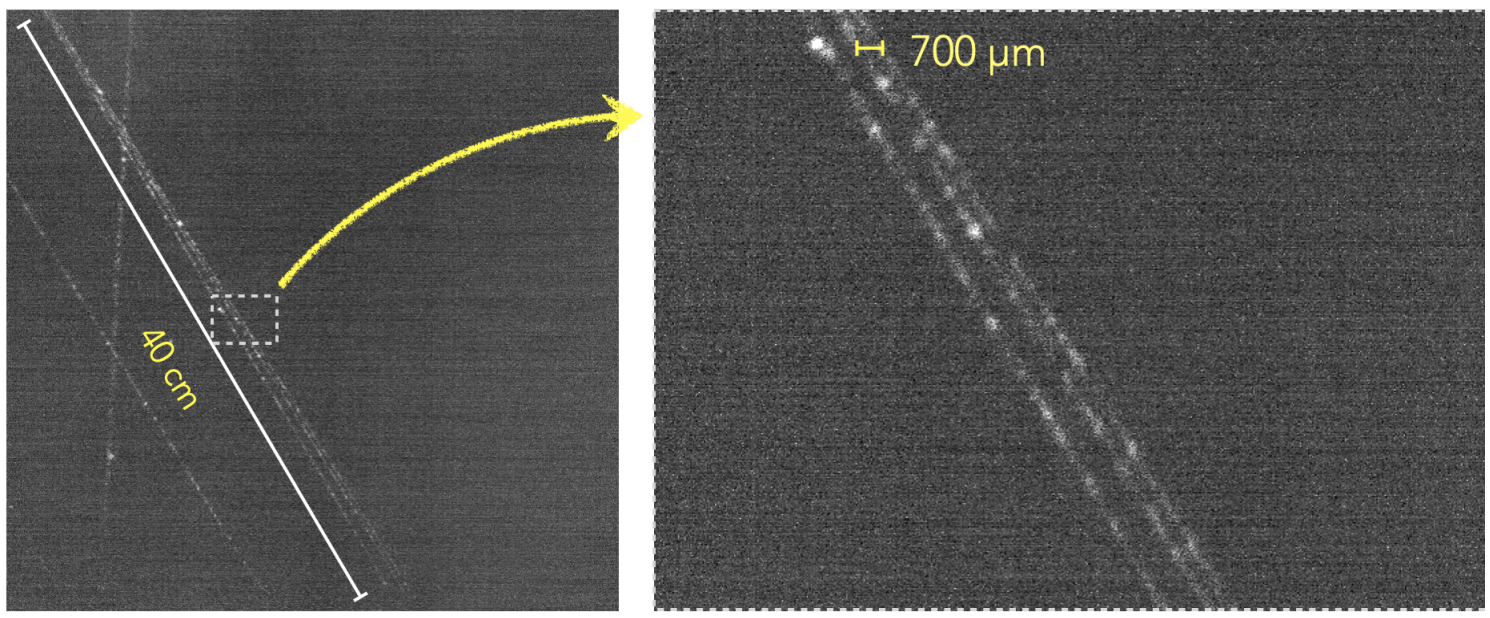

Figure 1: sCMOS camera image of few cosmic ray tracks crossing a TPC gas volume. Cosmic rays leave trails of ionization sites which are almost individually visible. A relative large region of about $30 \times 30 \mathrm{~cm}^{2}$ can be instrumented with a single camera (left). Sub-mm position resolution can be achieved (right). 


\section{The Cygno key elements}

The Cygno experiment is studying gas TPC with a gas mixture based on $\mathrm{He}_{-} \mathrm{CF}_{4}$ 60:40 kept at atmospheric pressure. This provides a target mass of $1.6 \mathrm{~kg}$ per $1 \mathrm{~m}^{3}$. The presence of light atoms in the molecules would allow to extend the sensitivity of Cygno down to few GeV DM masses. The $\mathrm{CF}_{4}$ gas has a light emission spectrum well matched with $\mathrm{CMOS}$ camera while the $\mathrm{He}-\mathrm{CF}_{4}$ mixture offers a longitudinal (transverse) diffusion coefficient of 110 (95) $\frac{\mu m}{\sqrt{c m}}$ at $1 \mathrm{kV} / \mathrm{cm}$ (from a Garfield simulation [7]). Moreover simulations based on SRIM [6] predict relatively large quenching factor (QF) for a NR energy deposit (at $1 \mathrm{keV} \mathrm{He} \mathrm{QF} \mathrm{is} \mathrm{about} 40 \%$ in the Cygno gas mixture).

In Fig. 2 a sketch of the $1 \mathrm{~m}^{3}$ Cygno demonstrator elements is shown. A 3-dimensional position reconstruction is envisaged with a sCMOS camera used for the $x$ - $y$ coordinates - parallel to the anode - and fast PMTs for the $z$ coordinate - parallel to the drift motion of the ionization clusters. The pixel size at the anode plane detected by the camera is $165 \times 165 \mu \mathrm{m}^{2}$ - requiring about $100 \mathrm{M}$ pixels for a $1 \mathrm{~m}^{2}$ anode plane.

The design of the Cygno demonstrator is currently being finalized for an installation in the next coming years at INFN LNGS. A Geant4-based simulation of the environmental gamma and neutron background in the LNGS underground hall inducing a recoil in the sensitive volume was performed. It predicts that a shielding composed of internal $5 \mathrm{~cm} \mathrm{Cu}$ layer with an external $2 \mathrm{~m}$ thick water tank can reduce the number of ER background events to few thousand in one year in the [0-20] keV range. This is prior to any attempt to apply particle identification based on the Cygno TPC tracking capabilities. The same shielding is reducing neutron induced NR events to about 10 per year in the same range of energy. However, the radioactivity of the materials composing the detectors are predicted to be the dominant source of background. Largest contributors are the camera body, its lens and the GEMs. An effort to produce low radioactivity lenses and GEMs is being pursued.

\section{Prototypes}

In the last years several small size prototypes have beed operated with various particles sources, from ultra-relativistic electrons [8] to ${ }^{55} \mathrm{Fe} \mathrm{X}$-rays [9] to 1-10 $\mathrm{MeV}$ neutron from an Am-Be source. They demonstrated ER with energy down to $1 \mathrm{keV}$ can be reconstructed with high efficiency and with an energy resolution of about $15 \%$. A month long overground operation of a 7 liters prototype with no significant interruption demonstrated how the experimental approach is solid in view of accumulating long exposures [10]. Also an algorithm to combine fast (PMT) and slow (camera) sensors was developed for a combined 3D tracking.

In order to identify patterns of energy deposit due to both NR and ER a 2D cluster identification technique (based on DBSCAN and Geodesic active contour) was developed [11]. By exposing the prototypes to neutrons NR have been identified with a $40 \%$ efficiency and a $96 \%$ ER rejection at the energy of about $6 \mathrm{keV}_{e e}$ [12]. A last prototype is currently being commissioned. It will be installed at LNGS for an underground run for a first assessment of the level of radiopurity of the employed materials and to prove the capability of shielding environmental radiation (see Fig.3). A run with no shielding to monitor environmental neutrons is foreseen. 


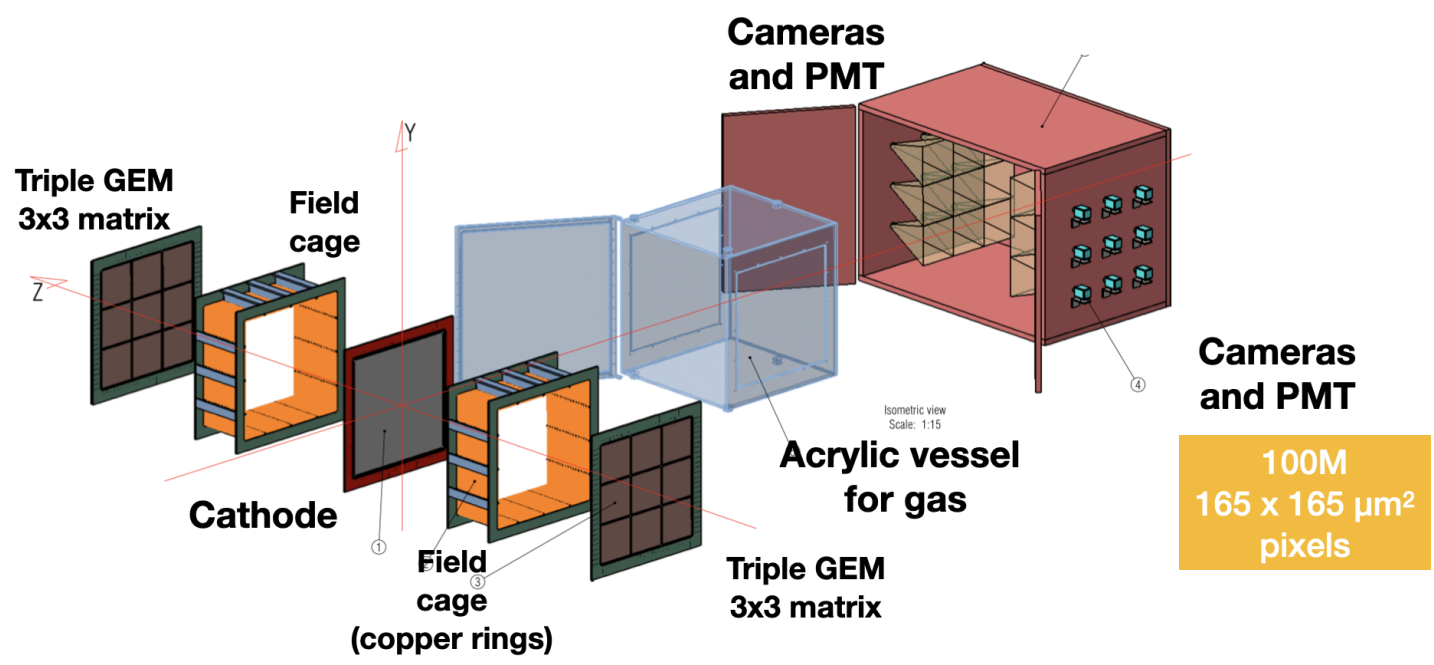

Figure 2: Conceptual design of a $1 \mathrm{~m}^{3}$ Cygno demonstrator, expanded in its own components.A transparent acrylic gas volume contains a field cage with two triple GEMs system at the anodes and with a central cathode. PMTs and cameras are installed outside the gas volume. The whole detector is then shielded with various layer of copper (or lead) and water (not shown in this picture).
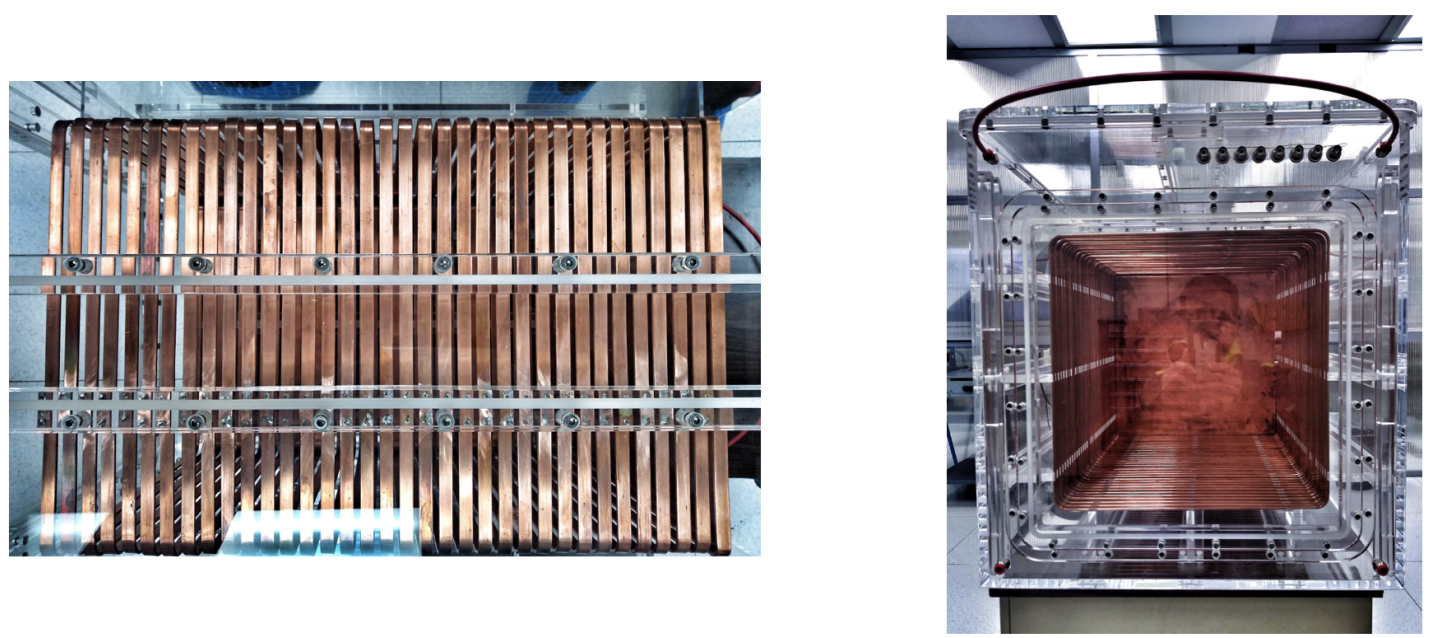

Figure 3: Images of the LIME prototype Cu field cage rings and its acrylic vessel. It has a $50 \mathrm{~cm}$ drift length corresponding to one of the 18 sector of the Cygno demonstrator.

\section{Cygno demonstrator sensitivity}

In order to evaluate the sensitivity to DM searches of the Cygno experiment a parametric simulation of the Cygno $1 \mathrm{~m}^{3}$ demonstrator was done including an angular resolution on the NR recoil direction of 30 degrees. In this simulation a perfect head-tail recognition and a standard halo model for the DM velocity distribution were assumed. Different scenarios for the total background 
events accepted by a $1 \mathrm{keV}$ energy threshold were considered. Exclusion curves are reported in Fig. 4.

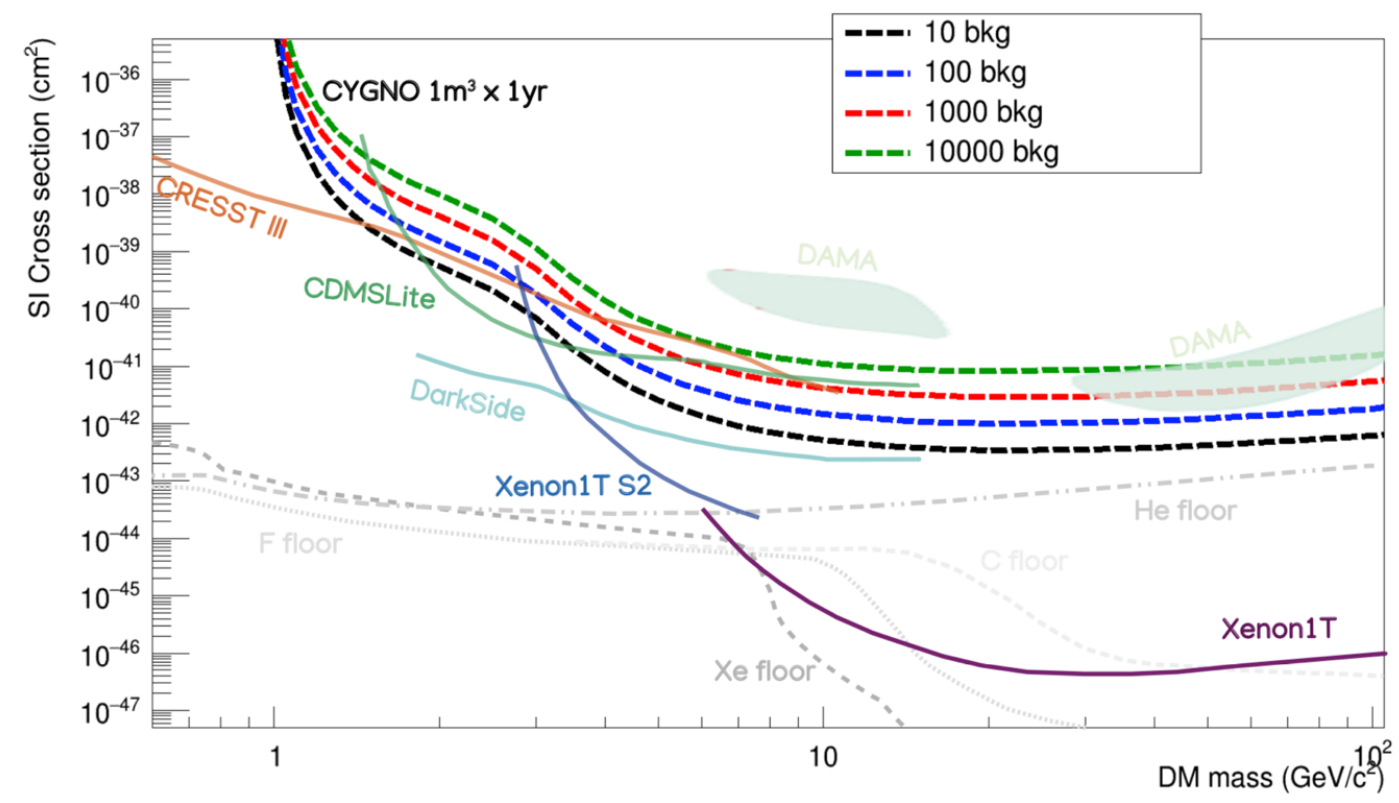

Figure 4: Exclusion curves for a spin independent dark matter search with a $1 \mathrm{~m}^{3}$ Cygno demonstrator in one year data-taking.

\section{Outlook}

The Cygno experiment aims to implement an optical readout for $\mathrm{He}-\mathrm{CF}_{4}$ TPC using GEM amplification and operated at atmospheric pressure. Promising results from a prototyping phase for ER and NR identification in the $\mathrm{keV}$ range are leading to the construction of a $1 \mathrm{~m}^{3}$ demonstrator to be operated underground at LNGS.

A number of $R \& D$ activities are currently pursued mostly to select the best material in terms of radiopurity. In parallel the study of electro-luminescence in the Cygno gas mixture [13] might lead to an increase of the light yield. Addition of an hydrogen -rich gas in the mixture might offer more sensitivity to GeV DM masses, while the operation of the gas TPC with negative ion drift (INITIUM project) might improve the track reconstruction.

The Cygno collaboration is envisaging a phase where a $30-100 \mathrm{~m}^{3}$ detectors could be built to study DM in the low mass DM range and with directional capabilities [14].

\section{Acknowledgements}

We want to thank General Services and Mechanical Workshops of Laboratori Nazionali di Frascati (LNF) and Laboratori Nazionali del Gran Sasso (LNGS) for their precious work and L. 
Leonzi (LNGS) for technical support. This project has received fundings under the European Union's Horizon 2020 research and innovation programme from the European Research Council (ERC) grant agreement No 818744. This project is supported by the Italian Ministry of Education, University and Research through the project PRIN: PROGETTI DI RICERCA DI RILEVANTE INTERESSE NAZIONALE “Zero Radioactivity in Future experiment” (Prot. 2017T54J9J)

\section{References}

[1] S. E. Vahsen, C. A. J. O’Hare, W. A. Lynch, N. J. C. Spooner, E. Baracchini, P. Barbeau, J. B. R. Battat, B. Crow, C. Deaconu and C. Eldridge, et al. "CYGNUS: Feasibility of a nuclear recoil observatory with directional sensitivity to dark matter and neutrinos," [arXiv:2008.12587 [physics.ins-det]].

[2] M. Marafini, V. Patera, D. Pinci, A. Sarti, A. Sciubba and E. Spiriti, "High granularity tracker based on a Triple-GEM optically read by a CMOS-based camera," JINST 10 (2015) no.12, P12010

[3] M. Marafini, V. Patera, D. Pinci, A. Sarti, A. Sciubba and E. Spiriti, "Optical readout of a triple-GEM detector by means of a CMOS sensor," Nucl. Instrum. Meth. A 824 (2016), $562-564$

[4] M. Marafini, V. Patera, D. Pinci, A. Sarti, A. Sciubba and E. Spiriti, "ORANGE: A high sensitivity particle tracker based on optically read out GEM," Nucl. Instrum. Meth. A $\mathbf{8 4 5}$ (2017), 285-288

[5] D. Pinci, E. Baracchini, G. Cavoto, E. Di Marco, M. Marafini, G. Mazzitelli, F. Renga, S. Tomassini and C. Voena, "High resolution TPC based on optically readout GEM," Nucl. Instrum. Meth. A 936 (2019), 453-455

[6] Ziegler, J. F., Ziegler, M. D., \& Biersack, J. P. 2010, Nuclear Instruments and Methods in Physics Research B, 268, 1818.

[7] R. Veenhof, 'GARFIELD, recent developments,” Nucl. Instrum. Meth. A 419 (1998), 726-730

[8] V. C. Antochi, et al. "Performance of an optically read out time projection chamber with ultra-relativistic electrons," Nucl. Instrum. Meth. A 999, 165209 (2021)

[9] V. C. Antochi, E. Baracchini, G. Cavoto, E. D. Marco, M. Marafini, G. Mazzitelli, D. Pinci, F. Renga, S. Tomassini and C. Voena, "Combined readout of a triple-GEM detector," JINST 13, no.05, P05001 (2018)

[10] E. Baracchini, et al. "Stability and detection performance of a GEM-based Optical Readout TPC with $\mathrm{He} / \mathrm{CF}_{4}$ gas mixtures," JINST 15, no.10, P10001 (2020)

[11] E. Baracchini, et al. "A density-based clustering algorithm for the CYGNO data analysis," JINST 15, no.12, T12003 (2020) 
[12] E. Baracchini, et al. "Identification of low energy nuclear recoils in a gas time projection chamber with optical readout," Measur. Sci. Tech. 32, no.2, 025902 (2021)

[13] E. Baracchini, et al. "First evidence of luminescence in a $\mathrm{He} / \mathrm{CF}_{4}$ gas mixture induced by non-ionizing electrons," JINST 15, no.08, P08018 (2020)

[14] F. D. Amaro, et al. "Directional Dark Matter Searches with CYGNO," Particles 4, no.3, 343-353 (2021) 